\title{
CHARACTERIZATIONS OF CONTINUA IN WHICH CONNECTED SUBSETS ARE ARCWISE CONNECTED $\left({ }^{1}\right)$
}

\section{E. D. TYMCHATYN}

ABSTRACT. The purpose of this paper is to give several characterizations of the continua in which all connected subsets are arcwise connected. The methods used are those developed by B. Knaster and K. Kuratowski, G. T. Whyburn and the author. These methods depend on Bernstein's decomposition of a topologically complete metric space into totally imperfect sets and on Whyburn's theory of local cutpoints. Some properties of connected sets in finitely Suslinian spaces are obtained. Two questions raised by the author are answered. Several partial results of Whyburn are obtained as corollaries of the main result.

The continua in which all connected subsets are arcwise connected and the continua which contain no punctiform or totally imperfect connected set have a long history. See for example Kuratowski and Knaster [4], Whyburn [10]-[14], and Tymchatyn [8]. Whyburn characterized the continua which contain no punctiform and no totally imperfect connected set in [11]. In this paper we shall characterize the continua in which all connected subsets are arcwise connected. We shall obtain several of Whyburn's partial results as corollaries. We shall also give some relations among these three classes of continua.

1. Definitions and preliminaries. Our notation largely follows Whyburn's Analytic topology [9]. We shall collect here some definitions for the convenience of the reader. A continuum is a nondegenerate, compact, connected, metric space. A continuum is said to be

(i) hereditarily locally connected if each subcontinuum is locally connected;

(ii) finitely Suslinian if each sequence of pairwise disjoint subcontinua forms a null sequence, i.e. the diameters of the subcontinua converge to zero;

Received by the editors January 10, 1975.

AMS (MOS) subject classifications (1970). Primary 54F50. cutpoints.

Key words and phrases. Arcwise connected, hereditarily locally connected, continua,

(1) This research was supported in part by a grant from the National Research Council. 
(iii) regular if the continuum has a basis of open sets with finite boundaries;

(iv) in class A if every connected subset is arcwise connected;

(v) a dendrite if it is locally connected and contains no simple closed curve.

It is known (see $[9$, Chapter V], [7] and [8]) that (v) $\Rightarrow$ (iv) $\Rightarrow$ (iii) $\Rightarrow$ (ii) $\Rightarrow$ (i) and none of these implications can be reversed.

We shall use the following proposition. Its proof is an easy exercise.

Proposition 1.1. Let $(X, d)$ be a metric space and let $A \subset B \subset X$. If $A$ is closed and for each $\epsilon>0,\{x \in B \mid d(x, A) \geqslant \epsilon\}$ is closed, then $B$ is closed.

Let $C$ be a connected and locally connected space. A point $p \in C$ is said to be a cutpoint of $C$ if $C \backslash\{p\}$ is not connected. The point $p \in C$ is said to be a local cutpoint of $C$ if $p$ is a cutpoint of some connected neighbourhood of $p$. We let $L(C)$ denote the set of local cutpoints of $C$.

A set $K$ is said to be $\sigma$-compact if it is the union of a countable family of compact sets.

The following theorem with the additional hypothesis that $C$ be locally compact appears as an exercise in $[9$, p. 63].

THEOREM 1.2. If $C$ is a connected, locally connected, separable, metric space then $L(C)$ is o-compact.

Proof. Let $U$ be a countable base for $C$ such that each member of $U$ is connected and open. It is easy to check that $p \in L(C)$ if and only if $p$ is a cutpoint of some member of $U$. By $[9$, III.5.3] for each $U \in U$ the set of cutpoints of $U$ is $\sigma$-compact. Hence, $L(C)$ is $\sigma$-compact since it is the union of a countable family of $\sigma$-compact sets.

The following theorem generalizes to local cutpoints a theorem of Whyburn $[9$, III.1.54].

THEOREM 1.3. If $M$ is a connected and locally connected, separable, metric space and $C$ is a dense, connected and locally connected subset of $M$ then $L(M) \backslash L(C)$ is at most countable.

Proof. Just suppose that the theorem fails. Let $U$ be a countable base for $M$ such that each member of $U$ is connected and open. As in the proof of Theorem 1.2 there is a $U \in U$ such that uncountably many points of $L(M) \backslash L(C)$ are cutpoints of $U$. By $[9$, III.3.1] there is an uncountable subset $D$ of $L(M) \backslash L(C)$ such that every point of $D$ is of potential order at most two in $U$ relative to $D$. Since $U$ is open in $M$ every point of $D$ is of potential order at most 
two in $M$ relative to $D$. In particular, there exist $a, b \in D$ such that $M \backslash\{a, b\}$ is not connected. Since $C$ is dense in $M, C \backslash\{a, b\}$ is not connected. Since $C$ is connected we may assume $a \in C$. If $C \backslash\{b\}$ is not connected then $b$ is a cutpoint and hence a local cutpoint of $C$. If $C \backslash\{b\}$ is connected then $a$ is a local cutpoint of $C$ since $a$ disconnects the connected neighbourhood $C\{b\}$ of $a$ in $C$. This is a contradiction.

Let $C$ be a connected space and let $A \subset C$. We say that $A$ is a cutting of $C$ if $C \backslash A$ is not connected. It is known (see [6, p. 244]) that each cutting of a connected, locally connected, separable, metric space $C$ between $a$ and $b$ where $a, b \in C$ contains an irreducible cutting of $C$ between $a$ and $b$ and each irreducible cutting of $C$ between $a$ and $b$ is closed.

Lemma 1.4. Let $C$ be a connected, locally connected, metric space, let $a, b$ $\in C$ and let $A \subset C \backslash L(C)$. If $A$ is an irreducible cutting of $C$ between $a$ and $b$ then $A$ has no isolated points. In particular, if $C$ is topologically complete then $A$ contains a Cantor set.

Proof. Just suppose that $p$ is an isolated point of $A$. Then $A \backslash\{p\}$ is closed in $C$ since $A$ is closed in $C$. Since $A$ is an irreducible cutting of $C$ between $a$ and $b, A \backslash\{p\}$ is not a cutting of $C$ between $a$ and $b$. Since $C$ is locally connected there is a component $V$ of $C \backslash(A \backslash\{p\})$ such that $a, b \in V$. Since $p$ cuts the open set $V, p \in L(C)$.

LEMMA 1.5. Let $X$ be a hereditarily locally connected continuum and let $C$ be a connected and nondegenerate set in $X$. Then $L(C)$ is dense in $C$.

Proof. Let $x \in C$ and let $U$ be a neighbourhood of $x$ in $X$ such that $C$ is not contained in $U$. Let $y \in C \backslash U$. By [9, V.3.3] there is a neighbourhood $V$ of $\boldsymbol{x}$ in $X$ with countable boundary $\operatorname{Bd}(V)$ such that $V \cup \operatorname{Bd}(V) \subset U$.

By [9, V.2.5] $C$ is locally connected. Since $\operatorname{Bd}(V) \cap C$ disconnects $C$ between $x$ and $y$ an irreducible subset $D$ of $\operatorname{Bd}(V) \cap C$ disconnects $C$ between $x$ and $y$. Now, $D$ contains an isolated point of $D$ since otherwise $\operatorname{Bd}(V)$ would contain a Cantor set. Since $D$ is an irreducible cutting of $C$ between $x$ and $y$ every isolated point of $D$ is in $L(C)$. Hence, $L(C) \cap U$ is nonvoid and $L(C)$ is dense in C.

2. Finitely Suslinian continua. In this section we shall obtain several results concerning arcwise connectedness of sets in finitely Suslinian continua.

Let $X$ be a space and let $C \subset X$. If $x \in C$ the arc component of $x$ in $C$ is $\{y \in C \mid y=x$ or there is an arc in $C$ with endpoints $x$ and $y\}$. The set $C$ is said to be arcwise connected if it has precisely one arc component. 
Lemma 2.1. Let $X$ be a finitely Suslinian continuum and let $C$ be an arcwise connected set in $X$. If $A$ is a compact subset of $X$ such that $C \cap A$ is dense in $A$ then there is a continuum $B$ such that $A \subset B \subset C \cup A$.

Proof. Let $D=\left\{d_{0}, d_{1}, \ldots\right\}$ be a countable dense set in $A \cap C$. Let $A_{1}$ be an $\operatorname{arc}$ in $C$ with endpoints $d_{0}$ and $d_{1}$. By induction there exists a sequence of continua $A_{1} \subset A_{2} \subset \cdots$ in $C$ such that for each $i=1,2, \ldots,\left\{d_{0}, \ldots, d_{i}\right\} \subset$ $A_{i}$ and $A_{i+1} \backslash A_{i}$ is either empty or homeomorphic to a half-open interval in the real line. Let $B=A_{1} \cup A_{2} \cup \cdots$. Since $D$ is dense in $A$ and $B$ is connected and contains $D, B \cup A$ is connected.

Since $X$ is finitely Suslinian the sets $A_{i+1} \backslash A_{i}, i=1,2, \ldots$, form a null sequence. Hence, if $d$ is a metric for $X$ and $\epsilon>0$ there exists a natural number $n$ such that

$$
\{x \in A \cup B \mid d(x, A) \geqslant \epsilon\} \subset A_{n} .
$$

Thus, $\{x \in A \cup B \mid d(x, A) \geqslant \epsilon\}$ is compact and, hence, closed in $X$. The set $A$ is also closed in $X$ since it is compact. By Proposition 1.1, $A \cup B$ is closed in $X$. Thus, $A \cup B$ is a continuum such that $A \subset A \cup B \subset C \cup A$.

We have as a corollary to Lemma 2.1 the following result of Whyburn [10, p. 334].

COROllary 2.2 (Why BURN [10]). If $X$ is a finitely Suslinian continuum and $C \subset X$ then the arc components of $C$ are closed in $C$.

Proof. Let $K$ be an arc component of $C$ and let $x_{1}, x_{2}, \ldots$ be a sequence in $K$ which converges to $x$ in $C$. By Lemma 2.1 the compact set $\left\{x, x_{1}, x_{2}, \ldots\right\}$ $C B \subset K \cup\{x\}$ where $B$ is a continuum. Since $X$ is hereditarily locally connected $B$ is arcwise connected.

Lemma 2.3. Let $X$ be a finitely Suslinian continuum and let $C$ be a subset of $X$. Let $\sim$ be an equivalence relation on $C$ such that each equivalence class of $\sim$ is an arcwise connected and closed set in $C$. Then $\sim$ is an upper semicontinuous relation on $C$.

ProOF. Every sequence of equivalence classes of $\sim$ is a null sequence. Thus, $\sim$ is upper semicontinuous (see $[9$, p. 122]).

The next result answers affirmatively a question raised by the author in [8].

THEOREM 2.4. Let $X$ be a finitely Suslinian continuum and let $C$ be a connected set in $X$. If $K$ is a connected set in $L(C)$ then $K$ is arcwise connected.

Proof. As in the proof of Theorem 1.3, $K \backslash L(K)$ is at most countable. By 
Theorem 1.2, $L(K)$ is $\sigma$-compact and hence $K$ is $\sigma$-compact. By Theorem 3.2 in $[2], K$ is arcwise connected.

3. Characterizations of class A. If $X$ is a space and $A \subset C \subset X$ we let $\mathrm{Cl}_{C}(A)$ denote the closure in $C$ of $A$.

The next theorem contains the main results in this paper.

THEOREM 3.1. If $X$ is a finitely Suslinian continuum the following seven conditions are equivalent:

(a) $X$ is in class $\mathrm{A}$.

(b) If $C$ is a connected $G_{\delta}$ in $X$ then $L(C) \not \subset A_{1} \cup A_{2} \cup \cdots$ where the $A_{i}$ are pairwise disjoint, closed, nonempty subsets of $C$.

(c) If $C$ is a connected $G_{\delta}$ in $X$ and $x, y \in C$ then there is an arc $A \subset C$ such that $x, y \in A$ and $A \backslash L(C)$ is at most countable.

(d) If $C$ is a connected $G_{\delta}$ in $X, A_{1}, A_{2}, \ldots$ is a sequence of painwise disjoint, closed subsets of $C$, and $x \in A_{1}$ and $y \in A_{2}$ then a countable subset of $C \backslash\left(A_{1} \cup A_{2} \cup \cdots\right)$ separates $x$ and $y$ in $C$.

$\left(\mathrm{b}^{\prime}\right),\left(\mathrm{c}^{\prime}\right)$ and $\left(\mathrm{d}^{\prime}\right)$ are obtained from $(\mathrm{b}),(\mathrm{c})$, and $(\mathrm{d})$, respectively, by replacing the condition " $C$ is a connected $G_{\delta}$ " by " $C$ is a connected set".

Proof. It is clear that $\left(b^{\prime}\right) \Rightarrow(b),\left(c^{\prime}\right) \Rightarrow(c)$ and $\left(d^{\prime}\right) \Rightarrow(d)$.

(a) $\Rightarrow\left(b^{\prime}\right)$. We suppose that $\left(b^{\prime}\right)$ fails and show that (a) also fails. Let $C$ be a connected set in $X$ such that $L(C) \subset A_{1} \cup A_{2} \cup \cdots$ where the $A_{i}$ are pairwise disjoint, nonempty, closed sets in $C$. We may suppose without loss of generality that $C$ is dense in $X$. Let

$$
Y=\bigcap_{i \neq j}\left(X \backslash\left(\mathrm{Cl}_{X}\left(A_{i}\right) \cap \mathrm{Cl}_{X}\left(A_{j}\right)\right)\right)
$$

Since $Y$ is a $G_{\delta}$ in $X, Y$ is topologically complete. Since $C$ is a dense, connected subset of $Y, Y$ is connected.

By a theorem of F. Bernstein (see [4]) $Y=P \cup Q$ where neither $P$ nor $Q$ contains a Cantor set. Let

$$
Z=P \cup \mathrm{Cl}_{Y}\left(A_{1}\right) \cup \mathrm{Cl}_{Y}\left(A_{2}\right) \cup \cdots
$$

We shall prove that $Z$ is a connected set that is not arcwise connected.

Let $A \subset Y\left(A_{1} \cup A_{2} \cup \cdots\right)$ be a cutting of $Y$. Since $Y$ is completely normal we may suppose that $A$ is closed in $Y$. Since $C$ is dense in $Y$, we may suppose that $A \cap C$ is a cutting of $C$ between two points $a$ and $b$ in $C$. Since $C$ is locally connected (see [9, V.2.5]), $A \cap C$ contains a set $B$ that is an irreducible cutting of $C$ between $a$ and $b$. By Lemma $1.4 B$ has no isolated point. Thus, $\mathrm{Cl}_{Y}(B) \subset A$ is a perfect set in the topologically complete metric space $Y$. It fol- 
lows that $A$ contains a Cantor set and so $A \not \subset Q$. Thus, $Q$ does not separate $Z$ in $Y$ and $Z$ is connected.

Let $x \in A_{1}$ and let $y \in A_{2}$. Let $K$ be an arc in $Y$ with endpoints $x$ and $y$. The sets $\mathrm{Cl}_{Y}\left(A_{1}\right) \cap K, \mathrm{Cl}_{Y}\left(A_{2}\right) \cap K, \ldots$ are pairwise disjoint closed sets in $K$. By Sierpiński's Theorem

$$
M=K \backslash\left(\mathrm{Cl}_{Y}\left(A_{1}\right) \cup \mathrm{Cl}_{Y}\left(A_{2}\right) \cup \cdots\right)
$$

is uncountable. An uncountable $G_{\delta}$ in a topologically complete, separable, metric space contains a Cantor set. Thus, $M \not \subset P$ and so $K \not \subset Z$. Since $K$ was an arbitrary arc in $Y$ with endpoints $x$ and $y, Z$ is a connected set in $X$ which is not arcwise connected. We have proved that (a) also fails.

(b) $\Rightarrow$ (a). We suppose that (a) fails and prove that (b) also fails. Let $C$ be a connected set in $X$ that is not arcwise connected. Let $\sim$ be the equivalence relation on $C$ that decomposes $C$ into its arc components. By Corollary 2.2 and Lemma 2.3, is upper semicontinuous. Let $\pi: C \rightarrow C / \sim$ be the natural projection of $C$ onto the quotient space $C / \sim$.

By Theorem $1.2 L(C)=C_{1} \cup C_{2} \cup \cdots$ where the $C_{i}$ are compact sets. Let $A_{1,1}=\pi^{-1}\left(\pi\left(C_{1}\right)\right)$ and let $A_{1, j}$ be empty for each $j>1$. Let $n>1$ be a natural number. Then $\pi\left(C_{n}\right)$ is a compact metric space. We wish to show that $\pi\left(C_{n}\right)$ is also totally disconnected.

For each $x \in C_{n}, \pi^{-1}(\pi(x)) \cap C_{n}$ is a compact set. By Lemma 2.1 there is a continuum $K(x)$ in $\pi^{-1}(\pi(x))$ which contains $\pi^{-1}(\pi(x)) \cap C_{n}$. We may suppose without loss of generality that if $x, y \in C_{n}$ such that $\pi(x)=\pi(y)$ then $K(x)$ $=K(y)$. It follows that if $x, y \in C_{n}$ then the continua $K(x)$ and $K(y)$ are either equal or disjoint. Since $X$ is finitely Suslinian it follows by Proposition 1.1 that $G_{n}=\bigcup\left\{K(x) \mid x \in C_{n}\right\}$ is compact. The components of $G_{n}$ are precisely the sets $K(x)$ where $x \in C_{n}$. Thus, $\left.\pi\right|_{G_{n}}: G_{n} \rightarrow \pi\left(G_{n}\right)=\pi\left(C_{n}\right)$ is a monotone map which acts on $G_{n}$ by collapsing the components of $G_{n}$ to points. It follows that $\pi\left(G_{n}\right)=\pi\left(C_{n}\right)$ is totally disconnected.

The set $\pi\left(C_{n}\right) \backslash \pi\left(C_{1} \cup \cdots \cup C_{n-1}\right)$ is open in the compact metric totally disconnected space $\pi\left(C_{n}\right)$. Hence,

$$
\pi\left(C_{n}\right) \backslash \pi\left(C_{1} \cup \cdots \cup C_{n-1}\right)=C_{n, 1} \cup C_{n, 2} \cup \cdots
$$

where the $C_{n, i}$ are pairwise disjoint closed sets in $\pi\left(C_{n}\right)$. For each $i=1,2, \ldots$ let $A_{n, i}=\pi^{-1}\left(C_{n, i}\right)$. Then $L(C)$ is contained in the union of the pairwise disjoint sets $\left\{A_{i, j} \mid i, j \geqslant 1\right\}$. These sets are closed in $C$ since $\pi$ is continuous. By Lemma $1.5 L(C)$ is dense in $C$ so it is easy to ensure that infinitely many of the sets $\left(A_{i, j}\right)$ are nonvoid.

Let

$$
Y=\bigcap_{(i, j) \neq(m, n)}\left(\mathrm{Cl}_{X}(C) \backslash\left(\mathrm{Cl}_{X}\left(A_{i, j}\right) \cap \mathrm{Cl}_{X}\left(A_{m, n}\right)\right)\right)
$$


Then $Y$ is a $G_{\delta}$ in $X$. Since $C$ is a dense connected set in $Y, Y$ is connected. By Theorem 1.3 $L(Y) \backslash L(C)$ is at most countable. Hence, $L(Y)$ is contained in the union of the pairwise disjoint closed sets $\mathrm{Cl}_{Y}\left(A_{i, j}\right), i, j=1,2, \ldots$, together with a countable set. Thus, (b) also fails.

(c) $\Rightarrow(\mathrm{b})$. We suppose that (b) fails and prove that (c) also fails. Let $C$ be a connected $G_{\delta}$ in $X$ such that $L(C) \subset A_{1} \cup A_{2} \cup \cdots$ where the $A_{i}$ are pairwise disjoint, closed, nonempty subsets of $C$. Let $x \in A_{1}$ and let $y \in A_{2}$. If $A$ is any arc in $C$ such that $x, y \in A$ then $A \backslash\left(A_{1} \cup A_{2} \cup \cdots\right)$ is uncountable by Sierpiński's Theorem. Thus, (c) also fails.

$\left(b^{\prime}\right) \Rightarrow\left(c^{\prime}\right)$. We suppose that $\left(c^{\prime}\right)$ fails and prove that $\left(b^{\prime}\right)$ also fails. Let $C$ be a connected set in $X$ such that there exist $x, y \in C$ with the property that each arc in $C$ with endpoints $x$ and $y$ contains uncountably many points of $C \backslash L(C)$.

Let $\sim$ be the equivalence relation on $C$ obtained by setting $a \sim b$ if and only if $a=b$ or there is an $\operatorname{arc} A$ in $C$ with endpoints $a$ and $b$ such that $A \backslash L(C)$ is at most countable. As in the proof of Corollary 2.2 the equivalence classes of $\sim$ are closed in $C$. By Lemma $2.3 \sim$ is upper semicontinuous since the equivalence classes of $\sim$ are also arcwise connected.

We can now argue exactly as in the proof that $(b) \Rightarrow(a)$ to show that $\left(b^{\prime}\right)$ also fails.

(d) $\Rightarrow$ (b). We suppose that (b) fails and prove that (d) also fails. Let $C$ be a connected $G_{\delta}$ in $X$ such that $L(C) \subset A_{1} \cup A_{2} \cup \cdots$ where the $A_{i}$ are nonempty, pairwise disjoint closed sets in $C$. By Lemma 1.4 every cutting of the topologically complete space $C$ which misses $L(C)$ is uncountable. Thus, (d) also fails.

$\left(b^{\prime}\right) \Rightarrow\left(d^{\prime}\right)$. We suppose that $\left(d^{\prime}\right)$ fails and prove that $\left(b^{\prime}\right)$ also fails. Let $C$ be a connected subset of $X$ such that there exist pairwise disjoint sets $A_{1}, A_{2}, \ldots$ which are closed in $C$ and $x \in A_{1}, y \in A_{2}$ such that no countable subset of $C \backslash\left(A_{1} \cup A_{2} \cup \cdots\right)$ separates $x$ and $y$ in $C$. Let

$$
Y=\mathrm{Cl}_{X}(C) \backslash \bigcup_{i \neq j}\left(\mathrm{Cl}_{X}\left(A_{i}\right) \cap \mathrm{Cl}_{X}\left(A_{j}\right)\right)
$$

For each $i$ let $B_{i}=\mathrm{Cl}_{Y}\left(A_{i}\right)$. Then $Y$ is a connected $G_{\delta}$ in $X$ since $C$ is a dense connected set in $Y$. The sets $B_{i}$ are pairwise disjoint closed sets in $Y$ and no countable subset of $Y\left(B_{1} \cup B_{2} \cup \cdots\right)$ separates $x$ and $y$. Let $B=B_{1} \cup B_{2}$ $U \cdots$. We shall prove that there is a connected subset $E$ of $Y$ such that $x, y$ $\in E$ and $L(E) \backslash B$ is at most countable. Thus, ( $\left.\mathrm{b}^{\prime}\right)$ also fails.

We define by transfinite induction a nest of connected subsets $\left(E_{\alpha}\right)$ of $Y$ as follows: Let $E_{0}=Y$. Let $\alpha$ be a countable ordinal number. Suppose that for each ordinal number $n<\alpha, E_{n}$ has been defined to be a connected subset of $Y$ such that $x, y \in E_{n}$ and no countable subset of $E_{n} \backslash B$ separates $x$ and $y$ in $E_{n}$. 
If $n+1<\alpha$ then there exist $a_{n+1}, b_{n+1} \in E_{n}$ such that $E_{n+1}$ is the component of $E_{n} \backslash\left\{a_{n+1}, b_{n+1}\right\}$ that contains $x$. If $n$ is a limit ordinal then $E_{n}=$ $\bigcap_{m<n} E_{m}$. We suppose that for each $n<\alpha, L\left(E_{n}\right) \backslash B$ is uncountable.

Case 1. $\alpha$ is the successor of the ordinal number $m$. By assumption $L\left(E_{m}\right) \backslash B$ is uncountable. As in the proof of Theorem 1.3 there exist $a_{\alpha}, b_{\alpha} \in$ $E_{m} \backslash B$ such that $E_{m} \backslash\left\{a_{\alpha}, b_{\alpha}\right\}$ is not connected. Let $E_{\alpha}$ be the component of $E_{m} \backslash\left\{a_{\alpha}, b_{\alpha}\right\}$ that contains $x$ and $y$. Then no countable subset of $E_{\alpha} \backslash B$ separates $x$ and $y$ in $E$.

Case 2. $\alpha$ is a limit ordinal. Let $E_{\alpha}$ be the component of $\bigcap_{n<\alpha} E_{n}$ which contains $x$. We shall show that $y \in E_{\alpha}$ and no countable subset of $E_{\alpha} \backslash B$ separates $x$ and $y$ in $E_{\alpha}$.

Let $D$ be a countable subset of $E_{\alpha}$. Let $D^{\prime}=D \cup \cup_{n<\alpha}\left\{a_{n+1}, b_{n+1}\right\}$. Since $D^{\prime}$ is countable, $x$ and $y$ lie in the same component $F$ of $Y D^{\prime}$. Now, $F$ is a connected, locally connected, topologically complete, metric space. Hence, there is an arc $G$ in $F$ with endpoints $x$ and $y$. By induction $G \subset E_{n}$ for each $n$ $\leqslant \alpha$. Hence, $x$ and $y$ lie in the same component of $E_{\alpha} D$.

Since $X$ does not contain uncountably many pairwise disjoint arcs it follows that for some countable ordinal $\alpha, L\left(E_{\alpha}\right) \backslash B$ is at most countable. This completes the proof of the theorem.

We list a variety of conditions that may be satisfied by a finitely Suslinian continuum $X$.

(i) $X$ is in class A.

(ii) $C$ a connected $G_{\delta}$ in $X \Rightarrow L(C)$ has finitely many components.

(iii) $C$ a subcontinuum in $X \Rightarrow L(C)$ is connected.

(iv) $C$ a connected $G_{\delta}$ in $X \Rightarrow L(C)$ is connected.

(v) $C$ a connected $G_{\delta}$ in $X \Rightarrow L(C)$ meets every cutting of $C$.

(vi) $C$ a connected $G_{\delta}$ in $X \Rightarrow L(C)$ is arcwise connected.

(vii) $C$ a connected subset of $X \Rightarrow L(C)$ is connected.

(viii) For each $a, b \in X$ every irreducible cutting of $X$ between $a$ and $b$ is at most countable.

(ix) Every pair of separated connected sets in $X$ can be separated by a countable set.

(x) $a, b \in X \Rightarrow$ there exist at most countably many $\operatorname{arcs}$ in $X$ with endpoints $a$ and $b$.

(xi) $C$ a true cyclic element in $X \Rightarrow C \backslash L(C)$ is countable.

(xii) Every irreducible cutting of $X$ is finite.

(xiii) Every sequence of distinct simple closed curves in $X$ is a null sequence.

(xiv) $X$ is a dendrite.

In the following result we have listed some relations among the fourteen properties listed above. Neither the list of properties nor the set of relations 
among them is exhaustive. Whyburn proved that properties (xi) and (xiii) imply (i) in [12] and [14] respectively. The implication (viii) $\Rightarrow$ (i) answers a question that arose in connection with [8].

THEOREM 3.2. Let $X$ be a finitely Suslinian continuum. The following relations exist among the conditions (i)-(xiv) listed above.

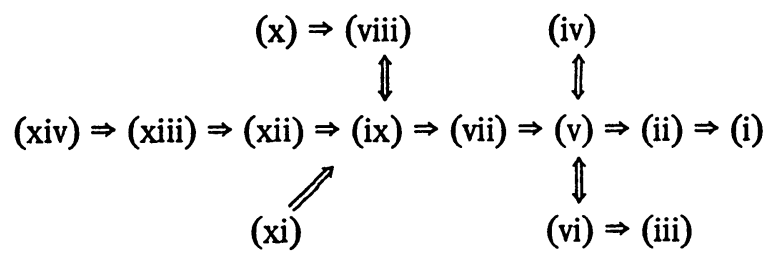

Proof. (ii) $\Rightarrow$ (i). Let $C$ be a connected $G_{\delta}$ in $X$. By Lemma $1.5 L(C)$ is dense in $C$. By Theorem 2.4 the components of $L(C)$ are arcwise connected. By Corollary 2.2 the arc components of $C$ are closed in $C$. Since $L(C)$ has only finitely many components $C$ has only finitely many arc components. Since $C$ is connected $C$ has only one arc component.

(iv) $\Rightarrow$ (vi) by Theorem 2.4 .

(viii) $\Rightarrow$ (vii) by Lemma 1.4 .

(xi) $\Rightarrow$ (viii) follows easily from $[9$, III.9.3]

(xiii) $\Rightarrow$ (xii). It is quite straightforward to prove that if $X$ satisfies (xiii) then every true cyclic element of $X$ is a finite graph.

All of the other implications are quite easy to see.

Question. Does (iii) $\Rightarrow$ (viii)? In particular does (iii) $\Rightarrow$ (i)?

It is known (see [6, p. 237]) that if $A$ and $B$ are regular continua and $A \cap$ $B$ is totally disconnected then $A \cup B$ is regular. The next example shows that class $\mathrm{A}$ does not have this property. This answers a question of A. Lelek.

EXAMPLE. There exists a plane regular continuum $X$ such that $X \subset D \cup E$ where $D$ is a dendrite, $E$ is in class $\mathrm{A}, D \cap E$ is a Cantor set and $X$ is not in class A.

Take $X=([0,1] \times\{0\}) \cup A_{1} \cup A_{2} \cup \cdots$ where the $A_{i}$ 's are defined inductively as follows: $A_{1}$ is the semicircle in the upper half-plane with center $(1 / 2,0)$ and radius $1 / 4$. For each $i \geqslant 2 A_{1}, A_{2}, \ldots, A_{i}$ are pairwise disjoint sets and $A_{i}$ is the union of $2 \cdot 3^{i-2}$ semicircles each of radius $1 /\left(2 \cdot 3^{i-2} \cdot 4^{i}\right)$. If $K$ is a semicircle in $A_{i}$ then the endpoints of $K$ are in $[0,1] \times\{0\}$ and the center of $K$ is an endpoint of some semicircle $L$ such that $L \subset A_{j}$ for some $j \in\{1, \ldots$, $i-1\}$.

Let $C=\mathrm{Cl}_{X}\left(A_{1} \cup A_{2} \cup \cdots\right) \cap([0,1] \times\{0\})$. Then $C$ is clearly a Cantor set. Let $B_{1}, B_{2}, \ldots$ be the closures of the components of $([0,1] \times\{0\}) \backslash C$. By construction $A_{i} \cap B_{j}$ is empty for all positive integers $i$ 
and $j$. It is easy to see that if $x \in L(X) \backslash\left(A_{1} \cup A_{2} \cup \cdots\right)$ then $x \in B_{i}$ for some i. Hence,

$$
L(X) \subset A_{1} \cup A_{2} \cup \cdots \cup B_{1} \cup B_{2} \cup \cdots .
$$

By Theorem 3.1, $X$ is not in class A.

Let $E$ be the intersection of $X$ with the closed lower half-plane. It is easy to see that for each connected set $F$ in $E, L(F)$ is connected. Hence, $E$ is in class A. Let $D^{\prime}=\mathrm{Cl}_{X}(X \backslash E)$. It is easy to construct a dendrite $D$ such that $D^{\prime} \subset D$ and $D$ is contained in the union of $C$ and the open upper half-plane.

4. Punctiform and totally imperfect connected sets. A set is said to be punctiform if it contains no nondegenerate continuum. In [11] Whyburn gave several characterizations of the locally connected continua which contain a punctiform connected set.

THEOREM 4.1 (Why BURN [11]). A locally connected continuum Y contains a punctiform connected set if and only if it contains a subcontinuum $D$ such that $L(D)$ is punctiform.

The following theorem relates class $\mathrm{A}$ to the continua which contain punctiform connected subsets.

Theorem 4.2. A finitely Suslinian continuum $X$ admits a monotone mapping onto a continuum $Y$ such that $Y$ contains a punctiform connected set if and only if there is a subcontinuum $C$ of $X$ such that $L(C) \subset A_{1} \cup A_{2} \cup \cdots$ where the $A_{i}$ are pairwise disjoint, nonempty compact sets.

Proof. $\Leftrightarrow$ Suppose $C$ is a subcontinuum of $X$ such that $L(C) \subset A_{1} \cup$ $A_{2} \cup \cdots$ where the $A_{i}$ are pairwise disjoint nonempty compact sets. Let $x \sim y$ in $X$ if and only if $x=y$ or $x$ and $y$ lie in some component of some $A_{i}$. By Lemma $2.3 \sim$ is an upper semicontinuous relation on $X$. Let $\pi: X \rightarrow X / \sim$ be the natural projection of $X$ onto the quotient space $X / \sim$. It is easy to see that $y \in \pi(C)$ is a local cutpoint of $\pi(C)$ only if $\pi^{-1}(y)$ is nondegenerate or meets $L(C)$. Since $\pi$ is a monotone map which identifies the components of the compact space $A_{i}$ to points, $\pi\left(A_{i}\right)$ is a totally disconnected compact metric space for each $i$. By the Sum Theorem for dimension zero $\pi\left(A_{1} \cup A_{2} \cup \cdots\right)$ is zero dimensional. Since $L(\pi(C)) \subset \pi\left(A_{1} \cup A_{2} \cup \cdots\right) \cup K$ where $K$ is a countable set, $L(\pi(C))$ is zero dimensional. By Theorem $4.1 \pi(X)$ contains a punctiform connected set.

$\Leftrightarrow$ Suppose $\pi: X \rightarrow Y$ is a monotone mapping of a finitely Suslinian continuum onto a continuum $Y$ such that $Y$ contains a connected, nondegenerate punctiform set. By Theorem 4.1 there is a continuum $D$ in $Y$ such that $L(D)$ is 
punctiform. As in the proof that (b) $\Rightarrow$ (a) in Theorem 3.1, $L(D)=B_{1} \cup B_{2}$ $\cup \cdots$ where the $B_{i}$ are pairwise disjoint compact sets. Since $L(D)$ is dense in $D$ by Lemma 1.5 , we may suppose that each $B_{i}$ is nonvoid.

Since $\pi$ is monotone and $D$ is a continuum in $Y, \pi^{-1}(D)$ is a continuum in $X$. If $x \in \pi^{-1}(D)$ such that $\pi^{-1}(\pi(x))=\{x\}$ and $x \in L\left(\pi^{-1}(D)\right)$ then $\pi(x) \in$ $L(D)$. For let $V$ be a neighbourhood of $x$ in $\pi^{-1}(D)$ such that $V \backslash\{x\}=P \cup Q$ where $P$ is separated from $Q$. If $\left(x_{i}\right)$ and $\left(y_{i}\right)$ are sequences in $P$ and $Q$ respectively which converge to $x$ then we have eventually $\pi\left(x_{i}\right) \neq \pi\left(y_{i}\right)$ since $\pi$ is monotone and $\pi^{-1}(\pi(x))=\{x\}$. Thus there is a closed neighbourhood $U$ of $x$ in $\pi^{-1}(D)$ such that $U \subset V$ and $\pi(U \cap P) \cap \pi(U \cap Q)=\{\pi(x)\}$. Since $\pi^{-1}(\pi(x))$ $=\{x\}$ it follows that $\pi(U)$ is a neighbourhood of $\pi(x)$ in $D$. Now, $\pi(U) \backslash \pi(x)=$ $\pi(U \cap P) \cup \pi(U \cap Q)$. Since $(U \cap P) \cup\{x\}$ and $(U \cap Q) \cup\{x\}$ are compact sets it follows that $\pi(U \cap P)$ and $\pi(U \cap Q)$ are separated sets. Hence, $\pi(x) \in$ $L(D)$.

Since $X$ is finitely Suslinian there are at most countably many $y \in D$ such that $\pi^{-1}(y)$ is a nondegenerate set. It follows that

$$
L\left(\pi^{-1}(D)\right) \subset \pi^{-1}(K) \cup \pi^{-1}\left(B_{1}\right) \cup \pi^{-1}\left(B_{2}\right) \cup \cdots
$$

where $K$ is a countable set in $D$.

COROLlARY 4.3. If $X$ is a finitely Suslinian continuum that is not regular then $X$ admits a monotone mapping onto a continuum which contains a punctiform connected set.

Proof. It was proved in [8] that $X$ is not in class $\mathrm{A}$ by proving that $X$ contains a subcontinuum $C$ such that $L(C) \subset A_{1} \cup A_{2} \cup \cdots$ where the $A_{i}$ are pairwise disjoint, nonempty, compact sets. The corollary now follows by Theorem 4.2.

A set is said to be totally imperfect if it contains no Cantor set. The following result (aithough it does not appear in the literature in precisely this form) is due to Whyburn [11].

TheOREM 4.4 (Why BURN [11]). A continuum $X$ contains a nondegenerate connected totally imperfect set if and only if it contains a subcontinuum $C$ such that $L(C)$ is at most countable.

THEOREM 4.5. A finitely Suslinian continuum $X$ admits a monotone mapping onto a continuum $Y$ such that $Y$ contains a totally imperfect connected set if and only if there is a subcontinuum $C$ of $X$ such that $L(C) \subset A_{1} \cup A_{2} \cup \cdots$ where the $A_{i}$ are pairwise disjoint nonempty subcontinua of $X$.

Proof. The proof is parallel to that of Theorem 4.2. 


\section{REFERENCES}

1. J. Dugundji, Topology, Allyn and Bacon, Boston, Mass., 1966. MR 33 \#1824.

2. J. Grispolakis, A. Lelek and E. D. Tymchatyn, Connected subsets of finitely Suslinian continua, Colloq. Math. (to appear).

3. W. Hurewicz and H. Wallman, Dimension theory, Princeton Math. Ser., vol. 4, Princeton Univ. Press, Princeton, N. J., 1941. MR 3, 312.

4. B. Knaster and $\mathrm{C}$. Kuratowski, A connected and connected im kleinen point set which contains no perfect subset, Bull. Amer. Math. Soc. 33 (1927), 106-109.

5. B. Knaster, A. Lelek and J. Mycielski, Sur les décompositions d'ensembles connexes, Colloq. Math. 6 (1958), 227-246. MR 21 \#6572.

6. K. Kuratowski, Topology, Vol. II, new ed., rev. and augmented, Academic Press, New York; PWN, Warsaw, 1968. MR 41 \#4467.

7. A. Lelek, On the topology of curves. II, Fund. Math. 70 (1971), 131-138. MR 44 \#995.

8. E. D. Tymchatyn, Continua in which all connected subsets are arcwise connected, Trans. Amer. Math. Soc. 205 (1975), 317-331.

9. G. T. Whyburn, Analytic topology, Amer. Math. Soc. Colloq. Publ., vol. 28, Amer. Math. Soc., Providence, R. I., 1942. MR 4, 86.

10. Concerning points of continuous curves defined by certain im kleinen properties, Math. Ann. 102 (1930), 313-336.

11. On the existence of totally imperfect and punctiform connected subsets in a given continuum, Amer. J. Math. 55 (1933), 146-152.

12. - Sets of local separating points of a continuum, Bull. Amer. Math. Soc. 39 (1933), 97-100.

13. Local separating points of continua, Monatsh. Math. Phys. 36 (1929), $305-314$.

14. - On a problem of W. L. Ayres, Fund. Math. 11 (1928), 296-301.

\section{DEPARTMENT OF MATHEMATICS, WAYNE STATE UNIVERSITY, DETROIT, MICHIGAN 48202}

Current address: Department of Mathematics, University of Saskatchewan, Saskatoon, Saskatchewan, Canada 